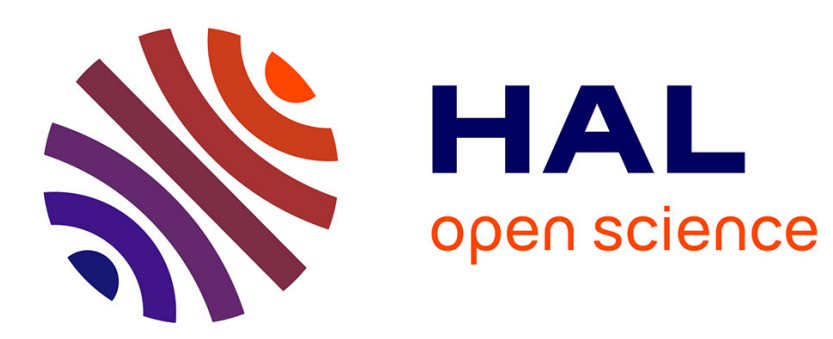

\title{
Minimum Convex-Cost Tension Problems on Series-Parallel Graphs
}

Bruno Bachelet, Philippe Mahey

\section{To cite this version:}

Bruno Bachelet, Philippe Mahey. Minimum Convex-Cost Tension Problems on Series-Parallel Graphs. RAIRO - Operations Research, 2003, 37 (4), pp.221-234. 10.1051/ro:2004202 . hal-00107129

\section{HAL Id: hal-00107129 \\ https://hal.science/hal-00107129}

Submitted on 16 Jan 2019

HAL is a multi-disciplinary open access archive for the deposit and dissemination of scientific research documents, whether they are published or not. The documents may come from teaching and research institutions in France or abroad, or from public or private research centers.
L'archive ouverte pluridisciplinaire HAL, est destinée au dépôt et à la diffusion de documents scientifiques de niveau recherche, publiés ou non, émanant des établissements d'enseignement et de recherche français ou étrangers, des laboratoires publics ou privés. 


\title{
Minimum Convex-Cost Tension Problems on Series-Parallel Graphs
}

\author{
Bruno Bachelet ${ }^{1}$ and Philippe Mahey ${ }^{2}$ \\ LIMOS, UMR 6158-CNRS, \\ Université Blaise-Pascal, BP 10125, 63173 Aubière, France.
}

Research Report LIMOS/RR03-06

${ }^{1}$ bachelet@ isima.fr - http://frog.isima.fr/bruno

${ }^{2}$ mahey@isima.fr 


\begin{abstract}
We present briefly some results we obtained with known methods to solve minimum cost tension problems, comparing their performance on non-specific graphs and on series-parallel graphs. These graphs are shown to be of interest to approximate many tension problems, like synchronization in hypermedia documents. We propose a new aggregation method to solve the minimum convex piecewise linear cost tension problem on series-parallel graphs in $O\left(\mathrm{~m}^{3}\right)$ operations.
\end{abstract}

Keywords: minimum cost tension, convex piecewise linear costs, series-parallel graphs.

\begin{abstract}
Résumé
Nous présentons brièvement quelques résultats que nous avons obtenus avec des méthodes connues pour résoudre des problèmes de tension de coût minimum, comparant leurs performances sur des graphes quelconques et sur des graphes série-parallèles. Il est montré que ces graphes sont intéressants dans l'approximation de problèmes de tension, comme la synchronisation de documents hypermédia. Nous proposons une nouvelle méthode d'agrégation pour résoudre le problème de la tension minimum avec des coûts convexes linéaires par morceaux sur des graphes série-parallèles en $O\left(\mathrm{~m}^{3}\right)$ opérations.
\end{abstract}

Mots clés : tension de coût minimum, coûts convexes linéaires par morceaux, graphes sérieparallèles.

\title{
Acknowledgements / Remerciements
}

This project was partially funded by France-Brazil cooperation project CAPES-COFECUB 398/02. 


\begin{abstract}
We present briefly some results we obtained with known methods to solve minimum cost tension problems, comparing their performance on non-specific graphs and on series-parallel graphs. These graphs are shown to be of interest to approximate many tension problems, like synchronization in hypermedia documents. We propose a new aggregation method to solve the minimum convex piecewise linear cost tension problem on series-parallel graphs in $O\left(\mathrm{~m}^{3}\right)$ operations.
\end{abstract}

Keywords: minimum cost tension, convex piecewise linear costs, series-parallel graphs.

\title{
Introduction
}

The exploding use of Internet and of hypermedia documents have turned crucial the necessity to dispose of robust on-line algorithms to manage complexity and interactivity. One of the resulting problems which has emerged recently is the synchronization of hypermedia documents by considering that each object can be compressed or delayed like an elastic spring. The heterogeneity of the objects that compose a hypermedia document turns their presentation in time and space a hard problem. On the other hand, interactivity means that real-time updates of the schedule of the document should be possible, increasing the need for faster decision-making algorithms.

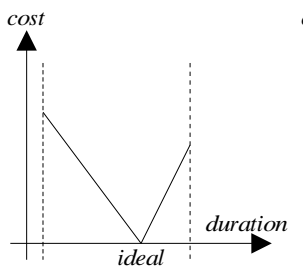

(a)

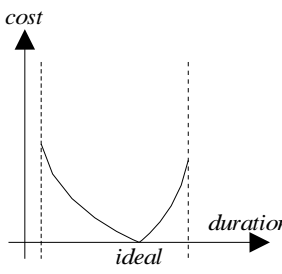

(b)

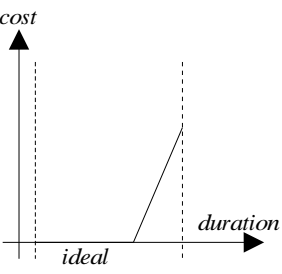

(c)

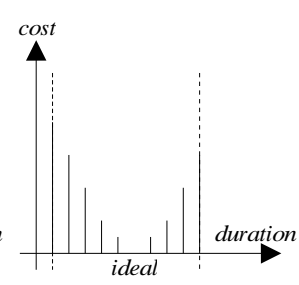

(d)

Figure 1: Examples of cost functions. a) Piecewise linear with a single ideal value. b) Non-linear, but convex and derivable. c) Piecewise linear with several ideal values. d) Discrete values.

As explained in [9] and [17], such documents are composed of media objects (audio, video, text, image...), which duration of presentation must be adjusted to satisfy a set of temporal constraints that express the progress of the animation as defined by the author. But for these constraints to be satisfied, the author must accept some flexibility on the duration (that we call ideal) of presentation of each object, pauses being totally forbidden if not explicitly wanted. To estimate the quality of an adjustment, a cost function, usually convex (cf. figure 1), is introduced for each object. To sum up, the problem we attempt to solve here is to find an adjustment of best quality, i.e. which minimizes the sum of the costs of the media objects.

The set of temporal constraints can be modeled as a directed graph $G=(X ; U)$ (cf. [6]) where $X$ is a set of nodes, $U$ a set of arcs, $m=|U|$ and $n=|X|$. The nodes represent events (the start or the end of presentation of an object). The arcs express duration constraints between nodes. With each arc $u$ is associated a time interval $\left[a_{u} ; b_{u}\right]$, an ideal duration $o_{u}$ and a cost function $c_{u}$ defined on the interval. An arc $u=(x ; y)$ between two nodes $x$ and $y$ means the event $x$ precedes $y$ and they are separated by a duration $\theta_{u}$ between $a_{u}$ and $b_{u}$, the ideal value being $o_{u}$. Figure 2 shows how to represent some of the main temporal relations used in hypermedia synchronization (introduced by [3]). 

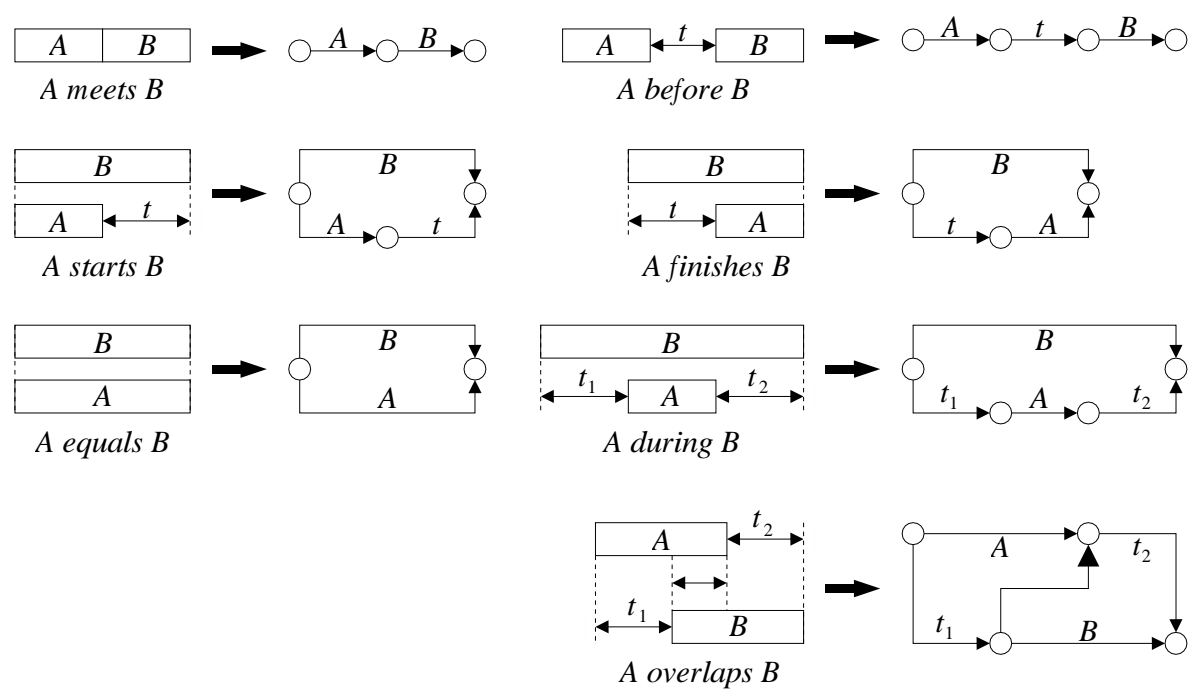

Figure 2: Graph representation of temporal constraints.

Let $\pi: X \mapsto \mathbb{R}$ be a potential function which assigns a date to each event node of the graph. Then the duration $\theta_{u}$ of an object associated with an arc $u=(x ; y)$ can be seen as the difference of potentials $\theta_{u}=\pi_{y}-\pi_{x}$, in other words, $\theta=\left(\theta_{u}\right)_{u \in U}$ is a tension vector on the graph (e.g. [7]). Denoting by $A$ the incidence matrix of the graph, i.e. matrix $A$ of dimension $(m \times n)$ with the elements $a_{x u}$ equal to -1 (if $u$ leaves $x$ ), +1 (if $u$ comes to $x$ ) or 0 (any other case), the problem is simply formulated as following:

$$
\left\{\begin{array}{l}
\operatorname{minimize} \sum_{u \in U} c_{u}\left(\theta_{u}\right) \\
\text { with } \theta=A^{T} \pi, a \leq \theta \leq b
\end{array}\right.
$$

And let $T_{G}$ be the set of feasible tensions, i.e. $T_{G}=\left\{\theta \in \mathbb{R}^{m} \mid \theta=A^{T} \pi, a \leq \theta \leq b\right\}$. In this article, we only consider convex two-piecewise linear cost functions as shown by figure 1a, the adaptation to more pieces of the methods described here is straightforward. Hence, from now on we consider the cost functions $c_{u}$ as following:

$$
c_{u}\left(\theta_{u}\right)=\left\{\begin{array}{l}
c_{u}^{1}\left(o_{u}-\theta_{u}\right), \text { if } \theta_{u}<o_{u} \\
c_{u}^{2}\left(\theta_{u}-o_{u}\right), \text { if } \theta_{u} \geq o_{u}
\end{array}\right.
$$

In section 1, we present the results we obtained with known methods to solve the minimum cost tension problem on graphs with non-specific structure. Then in section 2 , we recall and introduce some properties of the series-parallel graphs related to tension. Section 3 explains the aggregation method. Numerical results of this method and comparisons with the previous methods are presented and discussed in section 4. The last section ends this article with our first thoughts on how to exploit this method on non series-parallel graphs.

\section{Minimum Cost Tension Problem}

With convex piecewise linear costs, it is possible to model the problem with linear programs. It is a solution widely used in practice for the synchronization problem (e.g. [10], [17]). Another way to solve the problem is the out-of-kilter algorithm first introduced for the minimum cost flow 
problem [14] and then for the minimum cost tension problem in [18]. We present an adaptation of that method to piecewise linear costs in [5]. That algorithm is pseudo-polynomial, $O\left(m^{2}(A+B)\right)$ operations where $A=\max _{u \in U}\left\{a_{u} ; b_{u}\right\}$ and $B=\max _{u \in U}\left\{c_{u}^{1} ; c_{u}^{2}\right\}$. A polynomial method is presented in [15] but is only really efficient in practice for a special class of graphs (Penelope's graphs). More recently, [1] presents an algorithm to solve a more generic problem called the convex cost integer dual network flow problem, the algorithm consists in transforming the minimum cost tension problem into a minimum cost flow problem, solved with the well-known cost-scaling method (e.g. [2]). This algorithm is polynomial, $O\left(m n^{2} \log n A\right)$ operations, and proves to be very efficient in practice.

\begin{tabular}{|c|c|r|r|r|}
\hline Nodes & Arcs & CPLEX & Kilter & Cost-Scaling \\
\hline \hline 50 & 200 & 0,44 & 0,12 & 0,1 \\
50 & 400 & 0,83 & 0,3 & 0,19 \\
100 & 400 & 0,93 & 0,47 & 0,28 \\
100 & 800 & 2 & 1,3 & 0,54 \\
500 & 2000 & 12,5 & 15,4 & 3,5 \\
500 & 4000 & 37,7 & 49,1 & 6,8 \\
1000 & 4000 & 57,2 & 76,5 & 11,6 \\
1000 & 8000 & 193,7 & 239,9 & 20,4 \\
\hline
\end{tabular}

Table 1: Numerical results on non-specific graphs.

Table 1 aims at a practical comparison of the methods, which is always tricky because of all kinds of biases. But the goal here is to get an idea of how the methods behave on graphs with non-specific structure. Later in this article, we show the performance of these very same implementations on series-parallel graphs. Results are expressed in seconds, obtained on a RISC 6000 / $160 \mathrm{MHz}$ processor with an AIX Unix operating system. We use GNU C++ 2.95 compiler and its object-oriented features to implement the methods. For the linear programming, we use the simplex method provided in CPLEX 6.0 software. These results are the means of series of 10 tests on randomly generated graphs. Both $A$ and $B$ are fixed to 1000 . The implementation of the methods and the generation of the graphs are available in [4].

\section{Series-Parallel Graphs}

A common definition of series-parallel graphs is based on a recursive construction of the graphs (e.g. [12], [13], [22]) that is very intuitive and close to the way synchronization constraints are built in a hypermedia document.

A graph is series-parallel, also called SP-graph, if it is obtained from a graph with only two nodes linked by an arc, applying recursively the two following operations:

- The series composition, applied upon an arc $u=(x ; y)$, creates a new node $z$ and replaces $u$ by two $\operatorname{arcs} u_{1}=(x ; z)$ and $u_{2}=(z ; y)$ (cf. figure $3 \mathrm{a}$ ). We call series the relation that binds $u_{1}$ and $u_{2}$ and note it $u_{1}+u_{2}$.

- The parallel composition, applied upon an arc $u=(x ; y)$, duplicates $u$ by creating a new one $v=(x ; y)$ (cf. figure 3b). We call parallel the relation that binds $u$ and $v$ and note it $u / / v$. 


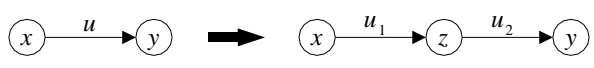

(a)

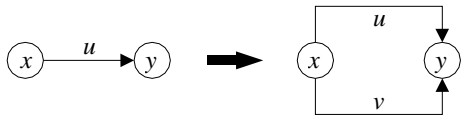

(b)

Figure 3: Series and parallel compositions.

We regroup the series and parallel relations under the term SP-relation. During the construction process, a SP-relation that binds two arcs can become a relation between two series-parallel subgraphs. Hence, we introduce the term single SP-relation to identify a SP-relation between two arcs. From the recursive definition of a SP-graph, it is easy to verify that a SP-graph has always a single SP-relation (the SP-relation created from the last composition). Hence, it is easy to check if a graph is series-parallel: find a single SP-relation in the graph, apply a reduction reverse to the composition that produces the SP-relation and go on again until only one arc remains in the graph. This linear-time method is explained in [22] and [20]. Another efficient approach to recognize a SP-graph is proposed in [13], based on the fact that paths in SP-graphs are organized a certain way.

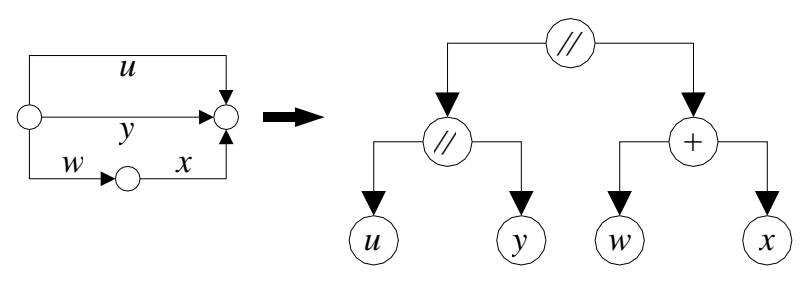

Figure 4: Example of SP-tree.

The SP-relations are binary operations, so we can represent a SP-graph by a binary tree called decomposition binary tree or SP-tree (cf. [22], [11]). Figure 4 shows a SP-tree of an SP-graph. All the algorithms cited earlier to recognize a SP-graph can be adapted, without any complexity loss, to build a SP-tree during their process. Hence we will use this representation to present our aggregation method.

From the definition of a SP-graph, it is obvious that a SP-graph has only one source node (i.e. without any predecessor) and only one target node (i.e. without any successor). Hence we define the main tension $\bar{\theta}$ of a graph as the tension between its source $s$ and target $t$, i.e. $\bar{\theta}=\pi_{t}-\pi_{s}$.

\section{Aggregation Method}

We present here the aggregation method to solve the minimum cost tension problem with convex piecewise linear cost functions (cf. figure 1a) on an SP-graph $G$. Note that the resolution of an optimization problem on this kind of graphs is usually easier than on non-specific graphs (e.g. [11], [8], [21]).

The aggregation method works on an SP-tree $T$ of the SP-graph $G$. The method is recursive: considering an SP-relation in $T$, it supposes that the optimal tensions of the two subgraphs implied in the relation are known, and from them it is possible to quickly build the optimal tension of the whole SP-relation. Hence, starting from the leaves of $T$, the optimal tension of each SP-relation is built to finally reach the root of the tree $T$.

To get an efficient algorithm, we need what we call the minimum cost function $C_{G}$ of a SPgraph $G$. This function represents the cost of the optimal tension where the main tension is forced to a given value. 


$$
C_{G}(x)=\min \left\{\sum_{u \in U} c_{u}\left(\theta_{u}\right) \mid \theta \in T_{G}, \bar{\theta}=x\right\}
$$

As each function $c_{u}$ is convex, the minimum cost function is indeed convex (assuming that $C_{G}(x)=+\infty$ if no feasible tension exists such that the main tension is forced to $x$ ).

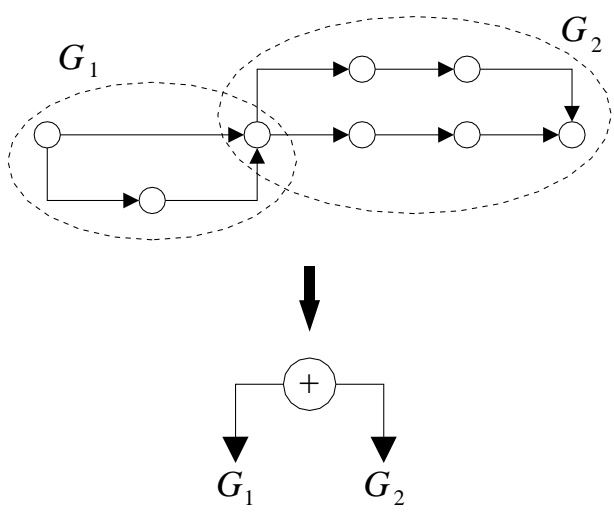

(a)

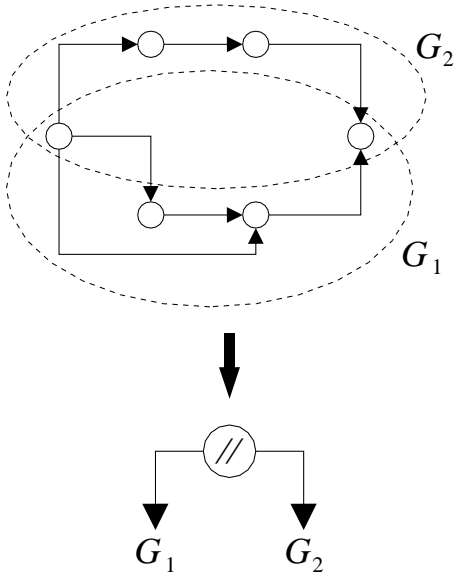

(b)

Figure 5: SP-relations between subgraphs.

We consider two series-parallel subgraphs $G_{1}$ and $G_{2}$, and suppose that their minimum cost functions $C_{G_{1}}$ and $C_{G_{2}}$ are known. If we look at the SP-relation $G_{1}+G_{2}$ (cf. figure 5a), $G_{1}$ and $G_{2}$ only share one node, hence there is no tension constraints between them. But if we add the constraint that the main tension of $G_{1}+G_{2}$ must be equal to $x$, it imposes to $x_{1}$ and $x_{2}$, the main tensions of $G_{1}$ and $G_{2}$, that $x=x_{1}+x_{2}$. Hence, the minimum cost function $C_{G_{1}+G_{2}}$ of the SP-relation $G_{1}+G_{2}$ is:

$$
C_{G_{1}+G_{2}}(x)=\min _{x=x_{1}+x_{2}} C_{G_{1}}\left(x_{1}\right)+C_{G_{2}}\left(x_{2}\right)
$$

It means $C_{G_{1}+G_{2}}$ is the inf-convolution $C_{G_{1}} \square C_{G_{2}}$. It is well-known that this operation maintains convexity (e.g. [19]).

If we look now at the SP-relation $G_{1} / / G_{2}$ (cf. figure $5 \mathrm{~b}$ ), $G_{1}$ and $G_{2}$ share their source and target nodes, hence the only tension constraint between them is that their main tensions $x_{1}$ and $x_{2}$ must be equal. If we add the constraint that the main tension of $G_{1} / / G_{2}$ must be $x$, then it imposes $x=x_{1}=x_{2}$. Hence, the minimum cost function $C_{G_{1} / / G_{2}}$ of the SP-relation $G_{1} / / G_{2}$ is:

$$
C_{G_{1} / / G_{2}}(x)=C_{G_{1}}(x)+C_{G_{2}}(x)
$$

It means $C_{G_{1} / / G_{2}}$ is simply the sum $C_{G_{1}}+C_{G_{2}}$, which is convex if $C_{G_{1}}$ and $C_{G_{2}}$ are convex. From our analysis of the two SP-relations, it is easy to write an algorithm that builds the minimum cost function $C_{G}$ of a SP-graph $G$. But what interests us is to find the minimum cost tension of $G$. We propose now a specific way to represent the minimum cost functions so we know not only the cost of the optimal tension of a SP-relation, but also how to build it.

For this purpose, we define the $t$-centered minimum cost function $C_{G}^{t}$ of $G$ as following:

$$
C_{G}^{t}(x)=C_{G}(x+t)-C_{G}(t)
$$


That means $C_{G}^{t}(0)=0$ and the function represents the minimum cost to increase or decrease the main tension from the value $t$.

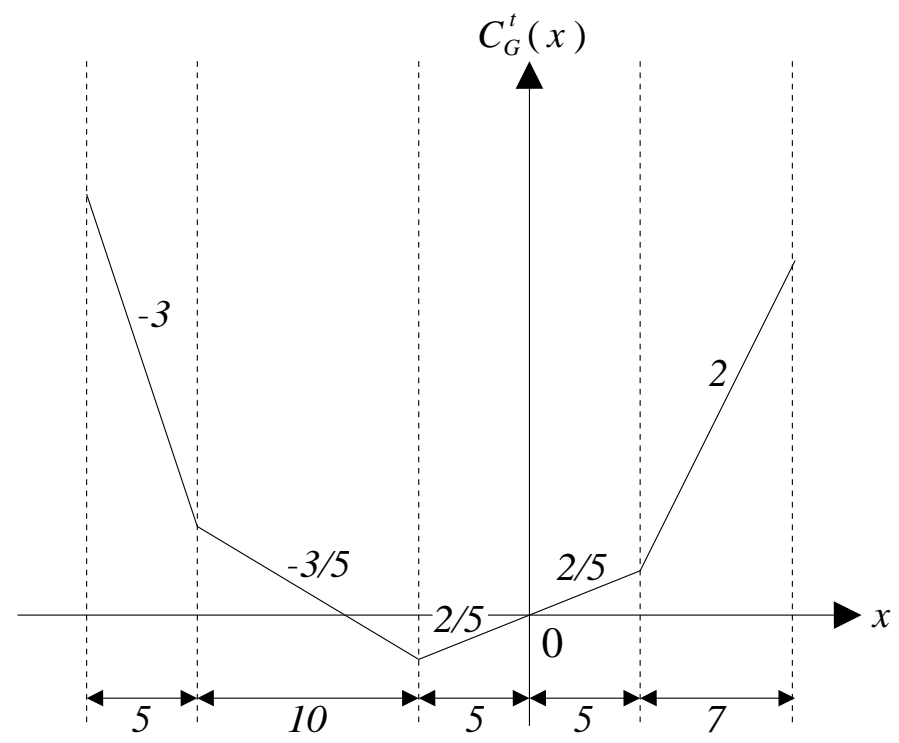

Figure 6: Example of $t$-centered minimum cost function.

We choose to represent this piecewise function with two sets $s h_{G}^{t}$ and $s t_{G}^{t}, s h_{G}^{t}$ represents $C_{G}^{t}$ on the interval $]-\infty ; 0\left[\right.$ and $s t_{G}^{t}$ represents $C_{G}^{t}$ on the interval $] 0 ;+\infty[$. These sets simply contain the definition of each piece of the function on the interval they represent. They contain triplets of the form $(c ; e ; l)$ where $c$ represents the slope of the curve, $e$ the length of the interval on which the piece is defined and $l$ is a set of the arcs that must be increased or decreased to adapt the tension on this piece. For efficiency reasons, the triplets are sorted from the smallest slope to the highest. Here are the sets $s t_{G}^{t}$ and $s h_{G}^{t}$ of the example of figure 6:

$$
\begin{aligned}
& s h_{G}^{t}=\{(-2 / 5 ; 5 ;\{a ; b\}) ;(3 / 5 ; 10 ;\{c\}) ;(3 ; 5 ;\{d ; e\})\} \\
& s t_{G}^{t}=\{(2 / 5 ; 5 ;\{a ; b\}) ;(2 ; 7 ;\{c ; e\})\}
\end{aligned}
$$

For instance, if we want to decrease the main tension of 1 unit, we need to decrease of 1 unit the tension of the arcs $a$ and $b$ and we will gain $2 / 5$ units.

Let us note $\theta_{G}^{*}$ the minimum cost tension of a graph $G$ and $C_{G}^{*}=C_{G}^{\overline{\theta_{G}^{*}}}$. We explain here how to find $\theta_{G}^{*}$ and build the $C_{G}^{*}$ function. First the $C_{u}^{*}$ function of an arc $u$ is represented by $s h_{u}^{*}=\left\{\left(c_{u}^{1} ; o_{u}-a_{u} ;\{u\}\right)\right\}$ and $s t_{u}^{*}=\left\{\left(c_{u}^{2} ; b_{u}-o_{u} ;\{u\}\right)\right\}$ with the optimal tension $\theta_{u}^{*}=o_{u}$.

Now consider the graph $G=G_{1}+G_{2}$ and suppose that we know the optimal tensions $\theta_{1}^{*}$ and $\theta_{2}^{*}$ and the minimum cost functions $C_{1}^{*}$ and $C_{2}^{*}$ of the subgraphs $G_{1}$ and $G_{2}$. The tension $\theta_{G}^{*}$ of $G$ made of the two tensions $\theta_{1}^{*}$ and $\theta_{2}^{*}$ is optimal, because there is no constraint between the two subgraphs after the series composition. To increase $\overline{\theta_{G}^{*}}$ we can choose to increase $\overline{\theta_{1}^{*}}$ or $\overline{\theta_{2}^{*}}$. If we look at $p_{1}=\left(c_{1} ; e_{1} ; l_{1}\right)$ and $p_{2}=\left(c_{2} ; e_{2} ; l_{2}\right)$, the first pieces of $s t_{1}^{*}$ and $s t_{2}^{*}$, we decide to increase $\overline{\theta_{1}^{*}}$ if $c_{1}<c_{2}$ or else $\overline{\theta_{2}^{*}}$. The same reasoning can be made to decrease $\overline{\theta_{G}^{*}}$. 


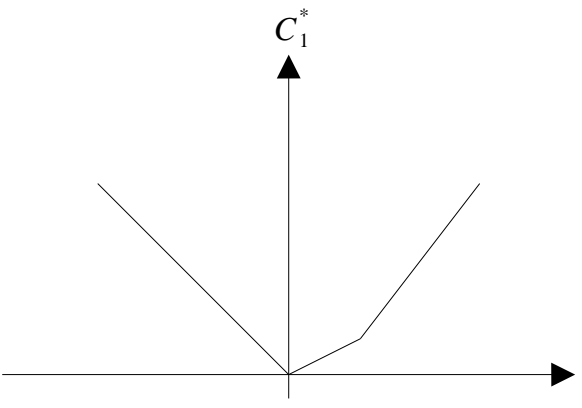

$$
\begin{aligned}
& s h_{1}^{*}=\{(1 ; 10 ;\{a ; b\})\} \\
& s t_{1}^{*}=\{(1 / 2 ; 4 ;\{b ; d\}) ;(4 / 3 ; 6 ;\{a\})\}
\end{aligned}
$$

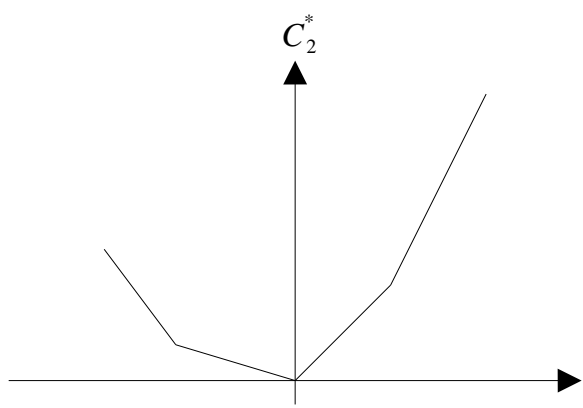

$s h_{2}^{*}=\{(1 / 3 ; 6 ;\{e\}) ;(5 / 4 ; 4 ;\{f ; g\})\}$

$s t_{2}^{*}=\{(1 ; 5 ;\{e ; g\}) ;(2 ; 5 ;\{f\})\}$

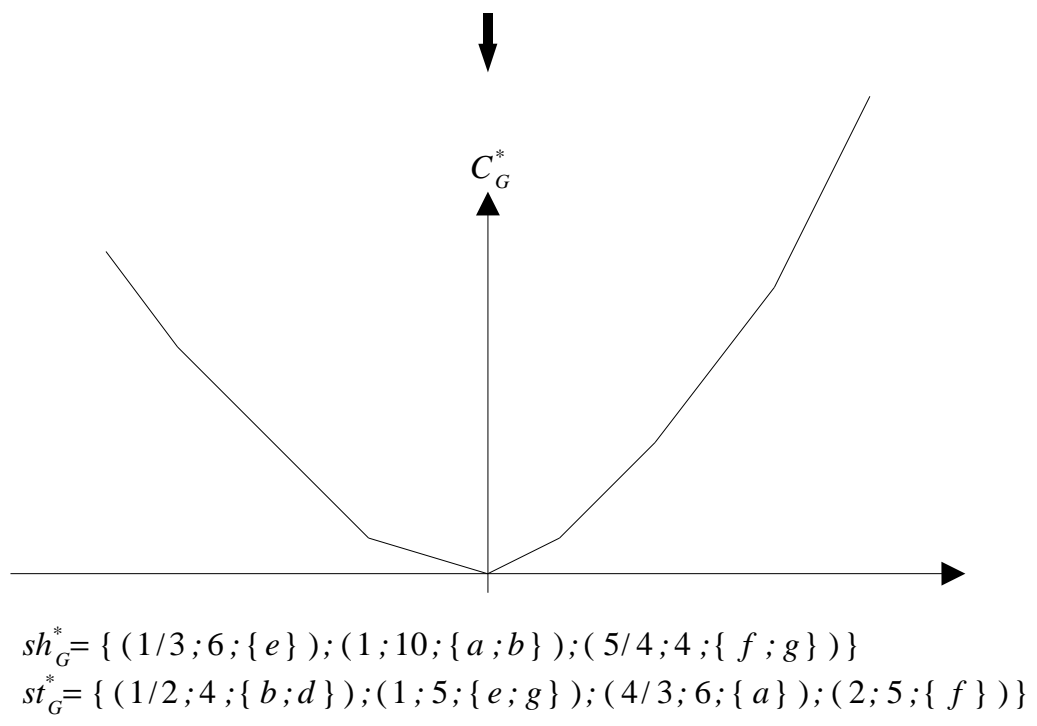

Figure 7: Example of minimum cost function of a series composition.

We can conclude that to build the function $C_{G}^{*}=C_{1}^{*} \square C_{2}^{*}$, we need to create $s h_{G}^{*}=s h_{1}^{*} \cup s h_{2}^{*}$ and $s t_{G}^{*}=s t_{1}^{*} \cup s t_{2}^{*}$ sorted from the smallest slope to the highest. Figure 7 shows an example. If we note $p_{1}$ and $p_{2}$ the numbers of pieces of $C_{1}^{*}$ and $C_{2}^{*}$, then $C_{G}^{*}$ has $p=p_{1}+p_{2}$ pieces, and the process of finding the optimal tension of a series composition needs $O(p m)$ operations $(O(p)$ operations to go through the $p$ pieces and $O(m)$ to copy a set of at most $m$ arcs for each piece).

We consider now the graph $G=G_{1} / / G_{2}$ and suppose that we know the optimal tensions $\theta_{1}^{*}$ and $\theta_{2}^{*}$ and the minimum cost functions $C_{1}^{*}$ and $C_{2}^{*}$ of the subgraphs $G_{1}$ and $G_{2}$. The parallel composition is possible only if $\overline{\theta_{1}^{*}}=\overline{\theta_{2}^{*}}$ (if we want to get a valid tension). As we need to find the optimal tension $\theta_{G}^{*}$ of the graph $G$, we need a method to equalize $\overline{\theta_{1}^{*}}$ and $\overline{\theta_{2}^{*}}$ optimally, i.e. such that the tension $\theta_{G}^{*}$ made of $\theta_{1}^{*}$ and $\theta_{2}^{*}$ is optimal. Suppose that $\overline{\theta_{1}^{*}}<\overline{\theta_{2}^{*}}$, to equalize $\overline{\theta_{1}^{*}}$ and $\overline{\theta_{2}^{*}}$ we can increase $\overline{\theta_{1}^{*}}$ and/or decrease $\overline{\theta_{2}^{*}}$, so we look at $p_{1}=\left(c_{1} ; e_{1} ; l_{1}\right)$ and $p_{2}=\left(c_{2} ; e_{2} ; l_{2}\right)$, the first pieces of $s t_{1}^{*}$ and $s h_{2}^{*}$. We decide then to increase $\overline{\theta_{1}^{*}}$ if $c_{1}<c_{2}$ or else to decrease $\overline{\theta_{2}^{*}}$. This process is repeated until $\overline{\theta_{1}^{*}}=\overline{\theta_{2}^{*}}$ (cf. algorithm 8 and figure 9 ). 


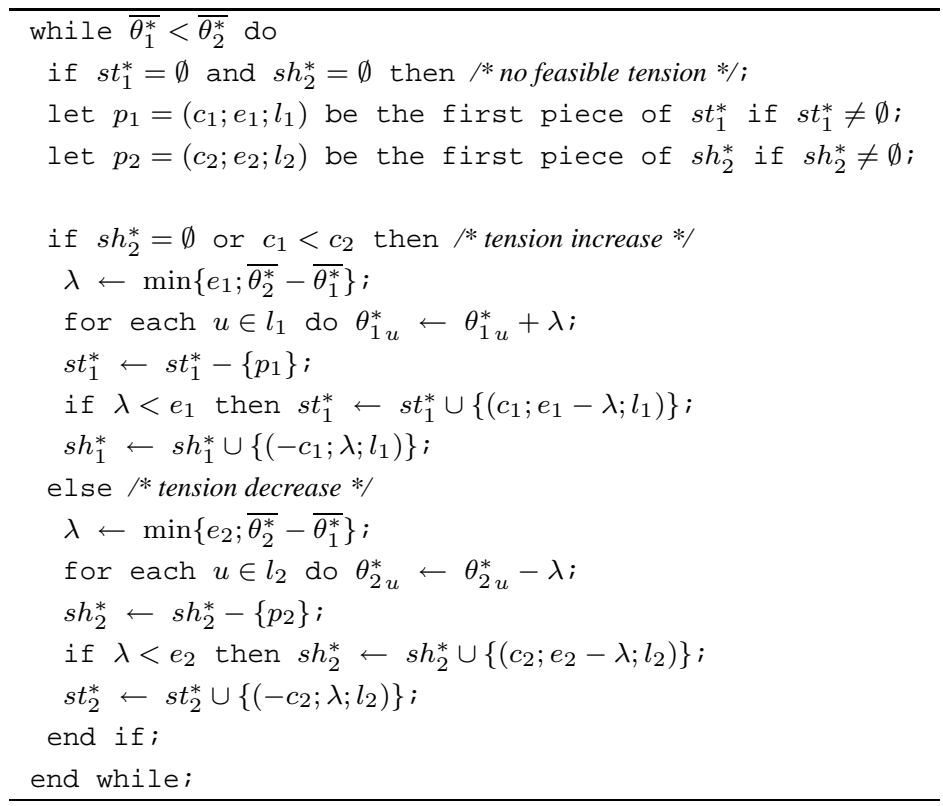

Figure 8: Algorithm to equalize parallel tensions.

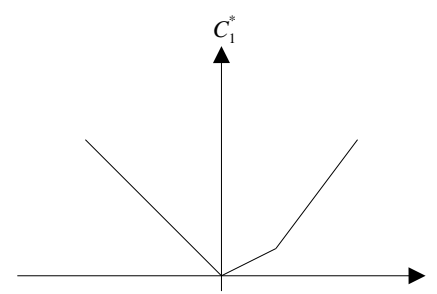

$s h_{1}^{*}=\{(1 ; 10 ;\{a ; b\})\}$

$s t_{1}^{*}=\{(1 / 2 ; 4 ;\{b ; d\}) ;(4 / 3 ; 6 ;\{a\})\}$ $\theta_{2}^{*}-\theta_{1}^{*}=8$

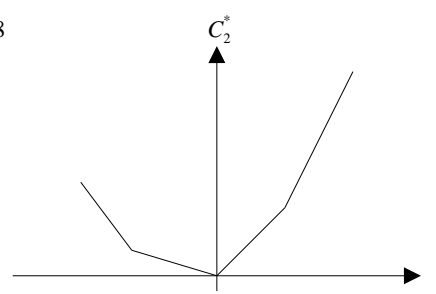

$s h_{2}^{*}=\{(1 / 3 ; 6 ;\{e\}) ;(5 / 4 ; 4 ;\{f ; g\})\}$ $s t_{2}^{*}=\{(1 ; 5 ;\{e ; g\}) ;(2 ; 5 ;\{f\})\}$

\section{1}

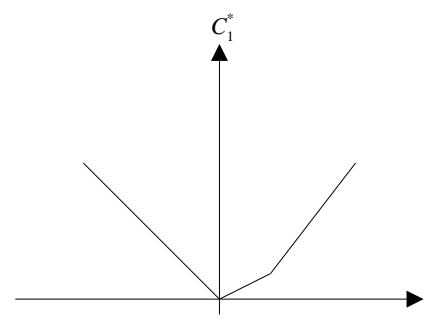

$s h_{1}^{*}=\{(1 ; 10 ;\{a ; b\})\}$ $s t_{1}^{*}=\{(1 / 2 ; 4 ;\{b ; d\}) ;(4 / 3 ; 6 ;\{a\})\}$ $\theta_{2}^{*}-\theta_{1}^{*}=2$

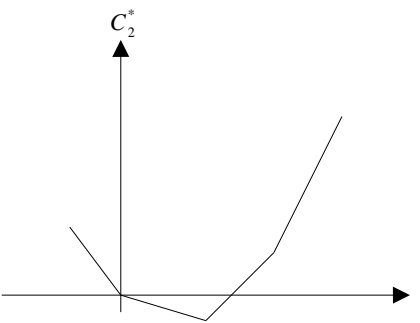

$s h_{2}^{*}=\{(5 / 4 ; 4 ;\{f ; g\})\}$

$s t_{2}^{*}=\{(-1 / 3 ; 6 ;\{e\}) ;(1 ; 5 ;\{e ; g\}) ;(2 ; 5 ;\{f\})\}$

\section{$\downarrow$}

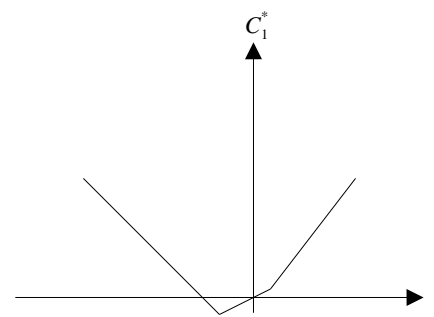

$s h_{1}^{*}=\{(-1 / 2 ; 2 ;\{b ; d\}) ;(1 ; 10 ;\{a ; b\})\}$ $s t_{1}^{*}=\{(1 / 2 ; 2 ;\{b ; d\}) ;(4 / 3 ; 6 ;\{a\})\}$ $\theta_{2}^{*}-\theta_{1}^{*}=0$

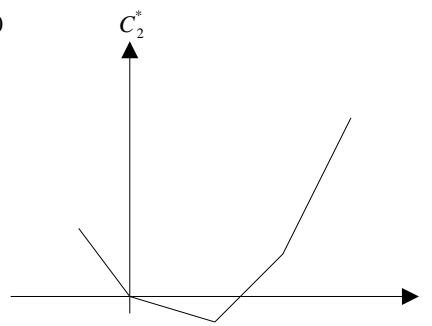

$s h_{2}^{*}=\{(5 / 4 ; 4 ;\{f ; g\})\}$

$s t_{2}^{*}=\{(-1 / 3 ; 6 ;\{e\}) ;(1 ; 5 ;\{e ; g\}) ;(2 ; 5 ;\{f\})\}$

Figure 9: Example of equalization of parallel tensions. 


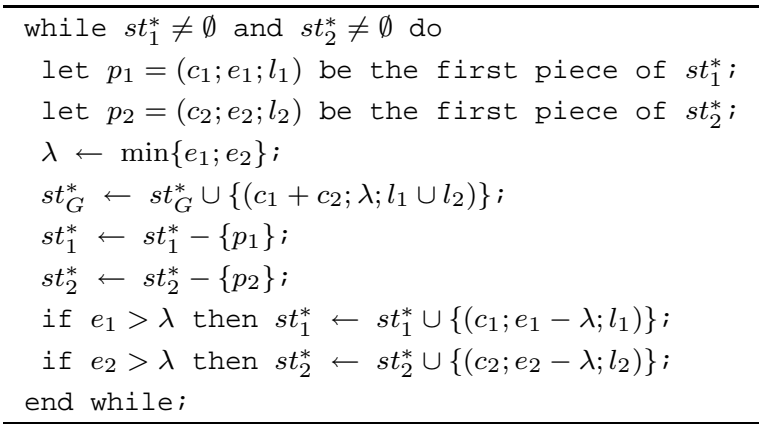

Figure 10: Algorithm to build the optimal tension of a parallel composition.

Then to build the function $C_{G}^{*}=C_{1}^{*}+C_{2}^{*}$ we use the procedure 10 (only detailed for the $s t_{G}^{*}$ part of the function) that is illustrated by figure 11. If we note $p_{1}$ and $p_{2}$ the numbers of pieces of $C_{1}^{*}$ and $C_{2}^{*}$, then $C_{G}^{*}$ has at most $p=p_{1}+p_{2}$ pieces, and the whole process of finding the optimal tension of a parallel composition needs $O(\mathrm{pm})$ operations (the equalization process go through at most $p$ pieces and copies at most $m$ arcs for each piece, the same for algorithm 10 that creates at most $p$ pieces and copies for each at most $m$ arcs).

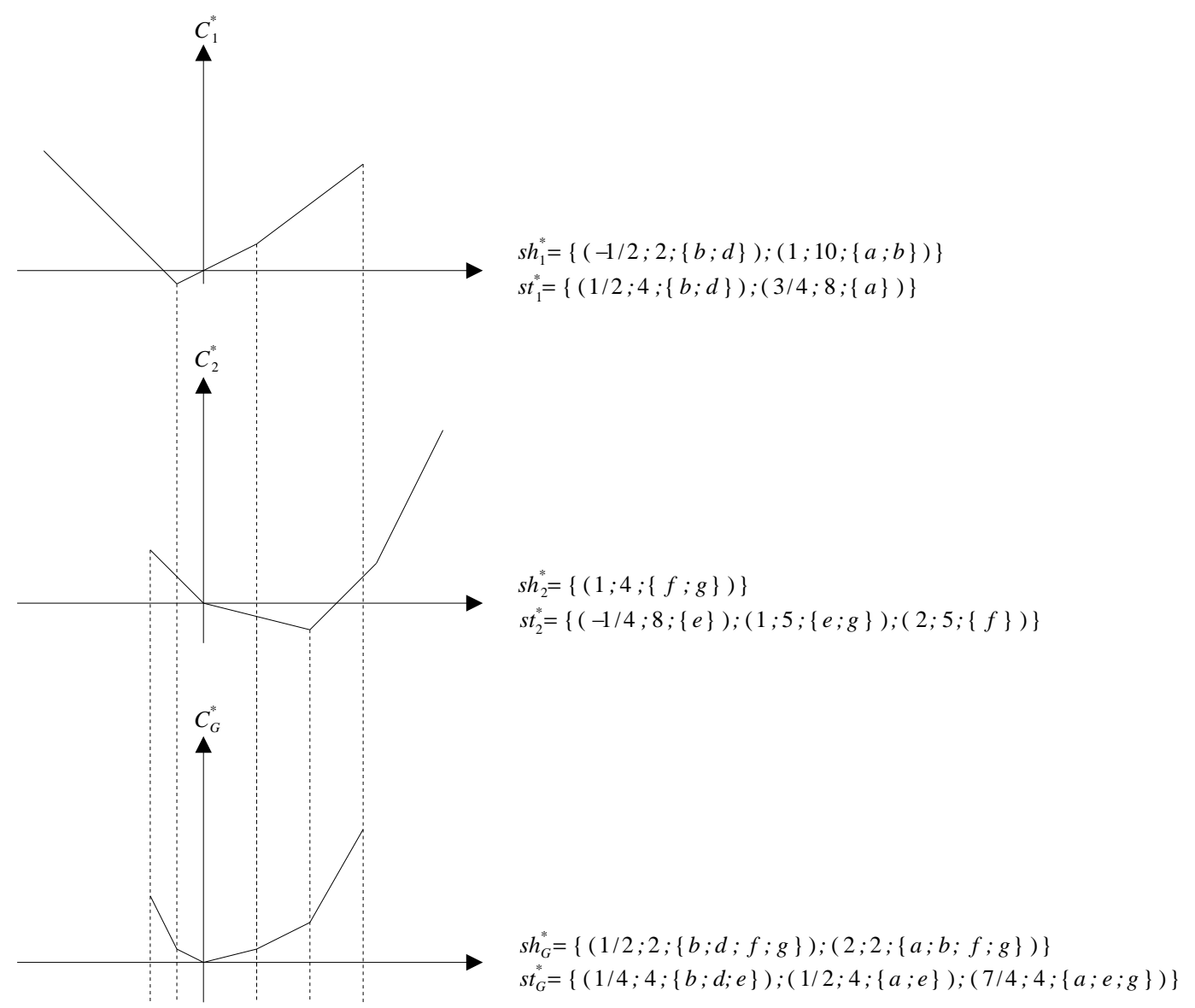

Figure 11: Example of minimum cost function of a parallel composition.

Finally algorithm 12 resumes the whole aggregation method, which, from the leaves of the SPtree of the graph, applies the series and parallel compositions with the construction of the optimal tension and the minimum cost function as we just explained in the previous paragraphs. 


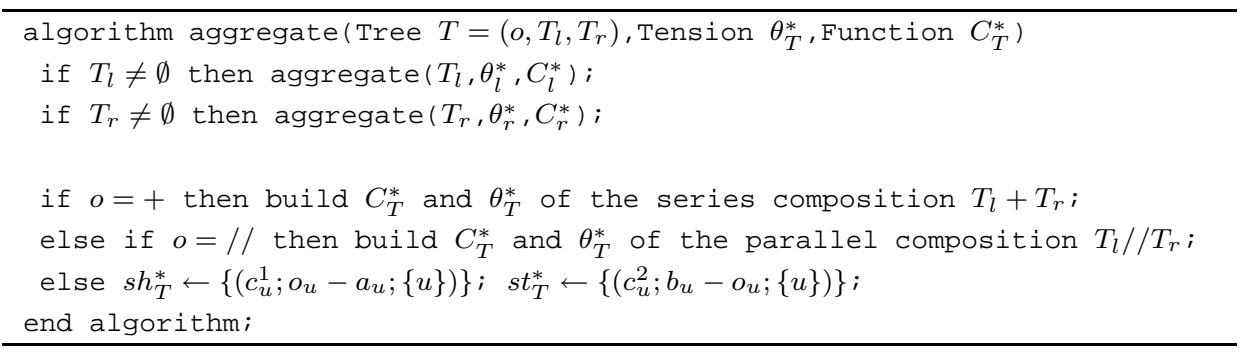

Figure 12: Algorithm to build the optimal tension of a SP-graph.

In this algorithm $T=\left(o ; T_{l} ; T_{r}\right)$ is the SP-tree with the root $o$, the left subtree $T_{l}$ and the right subtree $T_{r}$. We show now that this recursive method has a polynomial complexity.

Theorem 1 The aggregation algorithm performs $O\left(m^{3}\right)$ operations.

We established that each composition needs $O(p m)$ operations. It is known that a SP-graph contains $m-1$ SP-relations $(n-2$ series relations because each one creates a node and there are only two nodes at the beginning of the construction process, and $m-n+1$ parallel relations because any SP-relation creates an arc and there is only one arc at the beginning). So the aggregation needs $O\left(\mathrm{pm}^{2}\right)$ operations. We explained earlier that for each composition, if $p_{1}$ and $p_{2}$ are the numbers of pieces for $C_{1}^{*}$ and $C_{2}^{*}, C_{G}^{*}$ has at most $p_{1}+p_{2}$ pieces. That means if each arc has a two-piecewise cost function, the minimum cost function of the whole graph has at most $2 \mathrm{~m}$ pieces, and the aggregation needs $O\left(\mathrm{~m}^{3}\right)$ operations.

\section{Numerical Results}

Table 2 shows numerical results of the methods presented in section 1 and the aggregation algorithm on series-parallel graphs. Details on how the tests were performed can be found in the comments of table 1 (section 1). The linear programming and the out-of-kilter methods take advantage of the particular structure of the SP-graphs and behave really better on this class of graphs. However the cost-scaling approach on the dual of the problem does not work that well on this kind of instances, even with an improvement technique like the wave implementation (cf. [2]). The aggregation method reveals quite efficient, and not very sensitive to the graph dimension.

\begin{tabular}{|c|c|r|r|r|r|}
\hline Nodes & Arcs & CPLEX & Kilter & Cost-Scaling & Aggregation \\
\hline \hline 50 & 200 & 0,4 & 0,07 & 0,09 & 0,05 \\
50 & 400 & 0,71 & 0,15 & 0,2 & 0,09 \\
100 & 400 & 0,73 & 0,2 & 0,31 & 0,09 \\
100 & 800 & 1,4 & 0,38 & 0,63 & 0,18 \\
500 & 2000 & 4,4 & 3 & 4,5 & 0,5 \\
500 & 4000 & 10,7 & 5,3 & 11,3 & 0,99 \\
1000 & 4000 & 11,6 & 9 & 17,2 & 1 \\
1000 & 8000 & 30,8 & 21,5 & 34,5 & 2,1 \\
\hline
\end{tabular}

Table 2: Numerical results on series-parallel graphs.

\section{Conclusion}

We show here how to solve the minimum cost tension problem on series-parallel graphs with convex piecewise linear costs in $O\left(\mathrm{~m}^{3}\right)$ operations. But the real instances that interest us for the hypermedia synchronization are a bit more complex than the SP-graphs. They are indeed related 
to the generalized series-parallel graphs (cf. [16]). Through this article, we explained that, in the context of the minimum cost tension problem, a SP-graph can be reduced to a single arc with a convex piecewise linear cost function. One idea can be to identify series-parallel subgraphs of a non-specific graph, to aggregate these subgraphs and solve then the minimum cost tension problem on the reduced graph with any known method. To improve this simple idea, our future work will be to find algorithms to extract series-parallel components from a graph and to develop an efficient process (using the aggregation method) to find the minimum cost tension of the whole graph when assembling back these components.

\section{References}

[1] Ravindra K. Ahuja, Dorit S. Hochbaum, and James B. Orlin. Solving the Convex Cost Integer Dual Network Flow Problem. To appear, 1999.

[2] Ravindra K. Ahuja, Thomas L. Magnanti, and James B. Orlin. Network Flows - Theory, Algorithms, and Applications. Prentice Hall, 1993.

[3] James F. Allen. Maintaining Knowledge about Temporal Intervals. In Communications of the ACM, volume 26-11, pages 832-843, 1983.

[4] Bruno Bachelet. B++ Library. http://frog.isima.fr/bruno/?doc=bpp_library.

[5] Bruno Bachelet and Philippe Mahey. Optimisation de la présentation d'un document hypermédia. In Annales Scientifiques de l'Université Blaise Pascal, volume 110-42, pages 81-90, 2001.

[6] Bruno Bachelet, Philippe Mahey, Rogério Rodrigues, and Luiz Fernando Soares. Elastic Time Computation for Hypermedia Documents. In SBMidìa, pages 47-62, 2000.

[7] C. Berge and A. Ghoula-Houri. Programmes, jeux et réseaux de transport. Dunod, 1962.

[8] M.W. Bern, E.L. Lawler, and A.L. Wong. Linear-Time Computation of Optimal Subgraphs of Decomposable Graphs. In Journal of Algorithms, volume 8-2, pages 216-235, 1987.

[9] M. Cecelia Buchanan and Polle T. Zellweger. Specifying Temporal Behavior in Hypermedia Documents. In European Conference on Hypertext '92, pages 262-271, 1992.

[10] M. Cecelia Buchanan and Polle T. Zellweger. Automatically Generating Consistent Schedules for Multimedia Documents. In Multimedia Systems, pages 55-67. Springer-Verlag, 1993.

[11] Alak Kumar Datta and Ranjan Kumar Sen. An Efficient Scheme to Solve Two Problems for Two-Terminal Series Parallel Graphs. In Information Processing Letters, volume 71, pages 9-15. Elsevier Science, 1999.

[12] R.J. Duffin. Topology of Series-Parallel Networks. In Journal of Mathematical Analysis and Applications, volume 10, pages 303-318, 1965.

[13] David Eppstein. Parallel Recognition of Series-Parallel Graphs. In Information and Computation, volume 98-1, pages 41-55, 1992.

[14] D.R. Fulkerson. An Out-of-Kilter Method for Minimal Cost Flow Problems. In SIAM Journal on Applied Mathematics, volume 9, pages 18-27, 1961. 
[15] Malika Hadjiat. Penelope's Graph: a Hard Minimum Cost Tension Instance. In Theoretical Computer Science, volume 194, pages 207-218. Elsevier Science, 1998.

[16] Chin-Wen Ho, Sun-Yuan Hsieh, and Gen-Huey Chen. Parallel Decomposition of Generalized Series-Parallel Graphs. In Journal of Information Science and Engineering, volume 15-3, pages 407-417, 1999.

[17] Michelle Y. Kim and Junehwa Song. Multimedia Documents with Elastic Time. In Multimedia '95, pages 143-154, 1995.

[18] Jean-Marie Pla. An Out-of-Kilter Algorithm for Solving Minimum Cost Potential Problems. In Mathematical Programming, volume 1, pages 275-290, 1971.

[19] R.T. Rockefellar. Convex Analysis. Princeton University Press, 1970.

[20] Berry Schoenmakers. A New Algorithm for the Recognition of Series Parallel Graphs. Technical report, No CS-59504, Centrum voor Wiskunde en Informatica, Amsterdam, The Netherlands, 1995.

[21] K. Takamizawa, T. Nishizeki, and N. Saito. Linear-Time Computability of Combinatorial Problems on Series-Parallel Graphs. In Journal of the ACM, volume 29, pages 623-641, 1982.

[22] Jacobo Valdes, Robert E. Tarjan, and Eugène L. Lawler. The Recognition of Series Parallel Digraphs. In SIAM Journal on Computing, volume 11-2, pages 298-313, 1982. 